\title{
Brauchen wir mehr Spezialisierung in der Psychotherapie?
}

\author{
Ein Interview mit Mathias Berger
}

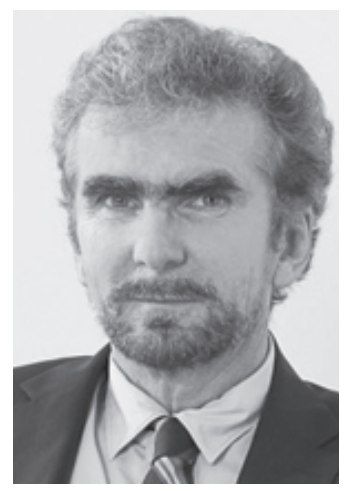

Wer sich heute auf die Suche nach einem für inn geeigneten Psychotherapeuten begibt, sei es aus primärer Intention oder auf Empfehlung seines Hausarztes, eines Facharztes oder einer Klinik, in der er aufgrund einer psychischen Erkrankung behandelt wurde, muss sehr häufig ein hohes Frustrationspotential vorweisen, denn einen freien Psychotherapieplatz zu finden, ist mitunter sehr schwierig. Besonders schwierig ist es in manchen ländlichen Regionen, wo die Suche nach einem Therapieplatz häufig enttäuschend verläuft. Eine in 2011 durchgeführte Studie der Universität Duisburg und der Deutschen Psychotherapeutenvereinigung (DPtV) zeigt, dass die Wartezeit auf einen Behandlungsplatz bei einem niedergelassenen Psychotherapeuten durchschnittlich knapp 80 Tage beträgt.

Noch schwieriger wird es, wenn es um die Spezialisierung für bestimmte Krankheitsbilder geht. Eine solche Spezialisierung ist sicherlich wünschens- und empfehlenswert und für den Therapieerfolg vieler Patienten von Vorteil; so gibt es Therapeuten, die beispielsweise eine große Anzahl von Patienten mit Borderline-Störungen, schweren Essstörungen oder schweren Zwangsstörungen behandeln. Jedoch sind diese Therapeuten oft ausgebucht, und in vielen Regionen des Landes verläuft die Suche nach solchen Spezialisten ergebnislos. Während in der somatischen Medizin vielfach eine zunehmende Spezialisierung für Krankheitsbilder eingetreten ist, scheint im Bereich der psychischen Erkrankungen das Denken in Schulen, Techniken oder Fachrichtungen immer noch stark vorhanden zu sein - eine aus der Sicht vieler Betroffener nicht immer befriedigende Situation.

Im vorliegenden Interview wurde Professor Mathias Berger, der als ärztlicher Direktor der Abteilung für Psychiatrie und Psychotherapie des Universitätsklinikums Freiburg die Einführung spezifischer Therapieangebote wie etwa der dialektisch-behavioralen Therapie der Borderline-Störungen oder der Cognitive behavioral anlysis system of psychotherapy (CBASP) stark gefördert hat, zu den Ursachen dieser Situation und seiner Perspektive für die Zukunft befragt.

Wenn man für einen Patienten einen ambulanten Psychotherapeuten sucht, ist es immer noch schwierig jemanden zu finden, der freie Kapazitäten hat. Besonders schwer wird es, wenn man für jemanden einen Psychotherapeuten sucht, der z.B. an einer Borderline-Störung, an einer Zwangsstörung, an einer Essstörung oder an einer anderen eher komplexen Störung leidet. Worin sehen Sie die Hauptursache für diesen massiven Versorgungsmangel?

\section{KARGER}

Fax +497614520714

Information@Karger.com

www.karger.com (c) 2013 S. Karger GmbH, Freiburg

Accessible online at:

www.karger.com/ver
Ein Hauptgrund liegt in der Ausbildung psychologischer und ärztlicher Psychotherapeuten. Viele Ausbildungsinstitute bieten zwar ein breites Spektrum an Krankheitsbildern für ihre Ausbildungsfälle an, Analysen zeigen jedoch, dass viele Psychotherapeuten oft vornehmlich Patienten mit Depressionen und Angststörungen unter Supervision zu behandeln lernen und eine breite klinische Ausbildung nicht mehr gewährleistet ist. In der Weiterbildung zu den Fachärzten für 
Psychiatrie und Psychotherapie bzw. psychosomatische Medizin und Psychotherapie ist in den Curricula nicht festgelegt, dass sie ein breites Spektrum von schwereren Erkrankungen unter Supervision psychotherapeutisch behandeln müssen. $\mathrm{Zu}$ der unzureichenden Qualifikation kommt das Problem der Richtlinienpsychotherapie mit häufig viel zu langen Behandlungsdauern. So behandelt ein niedergelassener ärztlicher oder psychologischer Psychotherapeut im Schnitt nur 50 Patienten im Jahr. Er wird sich in der Regel aus seiner Warteliste nicht gerade die schwersten und komplexesten Patienten heraussuchen, da es für die Therapie dieser Patientengruppe keinerlei finanziellen Anreiz gibt.

In der Weiterentwicklung der Therapie psychischer Erkrankungen, insbesondere in der Psychotherapie, war die Abkehr vom Denken in Schulen hin zu einem störungsspezifischen bzw. störungsorientierten Denken ein großer Fortschritt. Vielfach gibt es heute in psychiatrisch-psychotherapeutischen und auch psychosomatisch-psychotherapeutischen Kliniken Abteilungen bzw. Stationen, die sehr störungsorientiert, d.h. stärker spezialisiert und damit oft besonders effektiv arbeiten. Bräuchten wir nicht auch mehr störungsorientierte Psychotherapeuten, die auf bestimmte Krankheitsbilder spezialisiert sind wie z.B. den Borderline-Psychotherapeuten, den Trauma-Therapeuten, den Zwangs-Therapeuten usw? Wäre es nicht eine gute Vision für die Zukunft, wenn sich z.B. niedergelassene Psychotherapeuten auch nach außen hin als Spezialisten für bestimmte Störungen kennzeichnen könnten?

Dem stimme ich uneingeschränkt zu. Psychische Erkrankungen haben eine enorme Spannweite, die internistischen Erkrankungen in der Differenziertheit kaum nachstehen. Der hausärztliche Internist ist zwar ein Generalist, doch ist die Versorgung ohne Spezialisten für Kardiologie, Gastroenterologie, Rheumatologie, usw. nicht denkbar. In Analogie zu internistischen Erkrankungen ist unvorstellbar, dass ein Therapeut eine gleich hohe Kompetenz in der Behandlung aller psychischen Erkrankungen von Psychosen über Depressionen, Suchterkrankungen, Persönlichkeitsstörungen, Essstörungen, Aufmerksamkeitsdefizitstörungen usw. besitzt. Eine stärkere Spezialisierung der Psychotherapeuten auf bestimmte Krankheitsbilder würde die Versorgungssituation verbessern. Dafür wäre ein Abrücken vom bisherigen System der Richtlinienpsychotherapie sinnvoll. Dagegen bestehen aber massive ökonomische und ideologische Widerstände.

Sollte man für die Psychotherapie Zusatzzertifikate entwickeln wie etwa "psychotherapeutische Suchtbehandlung», "psychotherapeutische Schmerzbehandlung», usw?

In der Tat wäre eine solche Schwerpunktqualifikation, die auf dem Praxisschild erkennbar ist, für die Transparenz der Angebote relevant und ein wichtiger Baustein für eine partizipative Entscheidungsfindung. Im Moment ist die Suche nach einem qualifizierten Therapeuten für einen auf sich selbst gestellten Patienten mit einer Zwangsstörung, einer Bulimie oder einer Borderline-Persönlichkeitsstörung äußerst schwierig. Die Zugehörigkeit zu einer Schule wie Psychoanalyse, Tiefenpsychologie oder Verhaltenstherapie besagt sehr wenig über die spezifische Qualifikation des Therapeuten für unterschiedliche psychische Störungsbilder.

Die dritte Welle der Psychotherapie hat viele sinnvolle Weiterentwicklungen gebracht, aber im Gegensatz zur somatischen Medizin mit ihrer zunehmenden Spezialisierung auf Krankheitsbilder, scheint weniger eine Krankheitsspezialisierung, sondern mehr eine Methoden- und Technikspezialisierung eingetreten zu sein. "Eye Movement Desensitization and Reprocessing» $(E M D R)$-Therapeuten neigen dazu, die Methode eher breiter anzuwenden; Ähnliches geschieht für Schematherapie, Achtsamkeit, usw. Setzt sich das schulspezifische Denken damit fort?

Das ist in der Tat eine Gefahr der sogenannten dritten Welle der Verhaltenstherapie. Mit der Entideologisierung und Verwissenschaftlichung der Psychotherapie ist eine Entwicklung $\mathrm{zu}$ maßgeschneiderten Psychotherapieformen für bestimmte Störungsbilder erfolgt und beherrscht das Feld der randomisierten kontrollierten Studien und der evidenzbasierten Medizin. Eine Rückkehr zu Bildungen von Schulen mit dem Anspruch einer allgemeinen Wirksamkeit bei jedweder Form psychischer Erkrankungen ist im Moment unübersehbar. Die Begründer der «Acceptance and Commitment Therapy» (ACT) beispielsweise tendieren, wie auch viele Anhänger der «Mindfulness Based Therapy», zu einer Unterbewertung diagnostischer Zuordnungen.

Die große Zahl psychischer Störungsbilder und die häufige Komorbidität haben in den letzten Jahren deutlich werden lassen, dass der Ansatz der störungsspezifischen Psychotherapien nicht überspannt werden darf. Diese auf bestimmte Krankheitsbilder zugeschnittenen Therapieformen wie die Dialektisch-Behaviorale Therapie (DBT), die Interpersonelle Psychotherapie (IPT) oder das «Cognitive Behavioral Analysis System of Psychotherapy» (CBASP) setzen sich aus unterschiedlichen Modulen zusammen, die für die jeweiligen Störungsbilder als besonders geeignet erachtet werden und sich in randomisierten kontrollierten Studien als hilfreich erweisen. Wir müssen uns in Zukunft mehr an Therapiebausteinen orientieren, d.h. Modulen aus dem Gesamtspektrum psychotherapeutischer Methoden, die für bestimmte Symptomenkomplexe, wie etwa Impulskontrollstörungen, Suchtverhalten, Dissoziation, Suizidalität, Hoffnungslosigkeit oder Scham zur Verfügung stehen; wir müssen lernen, diese Bausteine zwar an Diagnosen zu orientieren, aber auch die individuellen Bedürfnisse und die komorbiden Aspekte des jeweiligen Krankheitsbildes berücksichtigend zusammenzustellen. In einer solchen individualisierten, modularen Psychotherapie spielen selbstverständlich Achtsamkeitsverfahren, Symptomakzeptanz oder Werteorientierung aus der ACT eine Rolle. 
Was ist Ihre Vision des Psychotherapeuten der Zukunft? Sollte er Medikamente verschreiben dürfen? Sollte er Arbeitsunfähigkeitsbescheinigungen ausstellen dürfen?

Die Konzeption einer Direktausbildung zum Psychotherapeuten scheint mir ein langer, kontroverser und steiniger Weg zu sein. Im Moment befürchte ich, dass diese Planungen dazu führen, dass die mehr als notwendige Korrektur der unsachlichen Degradierung von Diplom-Psychologen oder MasterPsychologen zu Studenten in Ausbildung ohne Anspruch auf eine tariflich festgelegte Bezahlung ihrer klinischen Tätigkeit auf die lange Bank geschoben wird. PiA müssen rechtlich und finanziell Ärzten in der Weiterbildung zum Facharzt gleichgestellt werden. Dann kann ihre klinische Tätigkeit auch wesentlich ausgedehnt und damit ihre Kompetenz in der Behandlung schwerer und komplexer Krankheitsbilder entscheidend verbessert werden. Bevor dieser nächste notwendige Schritt nicht erfolgt, halte ich es für kontraproduktiv, über einen neuen Beruf zwischen klinischer Psychologie und Psychiatrie/Psychosomatik weiter nachzudenken.

Ich danke Ihnen sehr für das Gespräch! 